\title{
Discussion: Analysis of multi-braced earth retaining structures
}

\author{
H. B. Xiao, J. Tang, Q. S. Li and Q. Z. Luo
}

\section{R. A. M. Watkins, FKW Ltd}

The authors have presented an interesting method for attacking the analysis of multi-braced earth retaining walls. As they have noted, analysis of this topic has become pertinent in many situations where relatively deep excavations are required. This occurs not only with deep basements but also with cut-andcover excavations for other structures, such as road and rail tunnels.

The method that they have developed is commendably concise and therefore allows for practical use in the initial stages of design. As they are aware, finite-element and finite-difference methods have been used over a number of years to model this problem, but the necessary stages whereby props or bracing are introduced have usually made the analysis more complicated than might otherwise be the case. Most recently, commercial software packages have adopted finite-difference or finiteelement methods to model the soil-structure interaction in two or three dimensions. However, a more concise method was presented by Bowles, ${ }^{11}$ which he described as a finite-element method but in fact only modelled the retaining structure as one-dimensional elements and modelled the soil as springs in a similar way to the method presented in this paper. Some time ago we were able to develop the program presented by Bowles to produce a stage-by-stage summation of deflections and member forces when excavation took place and props or struts were inserted. This provided a quick method for initial design purposes. A number of potential problems arise with this method, and it appears that similar problems may occur with the method presented by the authors.

The authors have adopted active earth pressures for the applied force above the excavation line but a constant earth pressure below that point. If this applied force is used and conventional values for the modulus of subgrade reaction are adopted, then the contributor has found that the deflected shape below the excavation line tends to overstate the deflection of the retaining structure when compared with deflection values measured on site by inclinometer. Have the authors obtained any site measurements of lateral pressures actually developed adjacent to the retaining structures that assist with this?

The method appears to assume that soil springs are required below the excavation line only. Where prestressed or jacked props are used, which is sometimes useful where the excavation is deep and the retaining structure is relatively flexible, the prop tends to compress the soil adjacent to the prop, and thus the earth pressure becomes larger than the active earth pressure by a variable amount. This would normally be modelled as a soil spring. A similar problem arises when a flexible retaining wall is used but the spacing of struts is variable. In some cases, if springs are used they have to be 'switched on' during one stage and then 'switched off' during other stages in the analysis. Have the authors considered how this problem can be addressed with their method?

It is often considered that the soil close to the excavation line has a modulus of subgrade reaction that is lower than the embedded soil (to deal with softening and/or disturbance), and clearly there are sites where the soil strata below the excavation line are quite variable. How do the authors deal with such variations in soil properties?

A difficulty also arises with the analysis when comparatively large deflections are found in the initial stages of design. Where such deflections occur, it may be assumed that the soil is no longer behaving as an elastic continuum. Potts has summarised some of the ways in which such elasto-plastic behaviour can be modelled. ${ }^{12}$ In reality the problem of large deflections may not be a practical problem for the initial design of retaining structures, where deflections generally need to be limited for surface deflections and the effects on adjacent structures and services. However, the need to model such behaviour becomes critical when back-analysing failures of retaining structures. Do the authors consider that the nonlinear behaviour of the soil needs to be modelled, and can they advise on how their proposed method can be adapted for this purpose?

Finally, as the authors appreciate, the shear forces and bending moments induced in the retaining wall depend not only on the deflections at various points but also on the slope of the deflected shape. Looked at in this context, the deflected shapes (calculated and measured) shown in their Figs 8 and 9 are not so similar. Do the authors have measured data, for instance from strain-gauging of the retaining structure, that would allow comparison between theoretical and actual bending moments and shear forces and the locations of the maxima?

\section{Authors' reply}

The lateral pressures acting on the retaining structure below the excavation line can be divided into two parts. The first part 
is the additional lateral pressure resulting from the excavation above the excavation line. The second part is the horizontal reaction force from the elastic soil, modelled by soil springs. It is noted that the additional lateral pressure is just the "constant earth pressure' mentioned by the discusser. Actually, if the excavation surface is considered as a surface of semi-infinite domain, and if the soil left above the excavation line is regarded as uniform overload acting on the excavation surface, then according to Boussinesq's theory ${ }^{13}$ the uniform overload will result in additional uniform lateral earth pressure in the semi-infinite domain. This is what was called "constant earth pressure'. We think it is proper to deal with it in such a way theoretically. Though we have not tested the lateral pressures adjacent to retaining structures on sites, we have conducted many site displacement measurements of retaining structures in Shanghai. Close agreement between our calculated and tested results has been found. Many researchers have proposed different methods to consider the lateral pressures acting on retaining structures below the excavation line. However, our experiences from some practical projects revealed that it is reasonable to adopt the proposed model to analyse soft, silty soil in the Shanghai area.

In our opinion, the lateral pressures and displacements are induced mainly from the remaining soil above the excavation line where prestressed or jacked props are not used. Therefore soil spring models are adopted below the excavation line to simplify the analysis procedure. Actually it is very effective to adjust internal forces and deformations of retaining structures by using prestressed props or struts with variable spacing. However, our comparative studies on many deep excavation projects have shown that executing large prestress forces may not be the most economical way to design a whole retaining structure. The retaining structure may fail when overloaded prestressed forces are applied to the props. In order to ensure the stability of a retaining structure, we have to increase the embedding depth of the retaining structure below the excavation line. The deeper the excavation is, the more embedding depth the retaining structure needs. If prestressed forces must be applied to props in some cases, soil springs are required below the excavation line. In this case, what we need to do is to increase the earth pressures above the excavation line according to the magnitudes of prestressed force. Therefore the lateral pressures acting on the retaining structure above the excavation line are the sum of the active earth pressures and the prestress forces. In this case it is not necessary to use soil springs above the excavation line. In other words, no such case exists in our method where the soil springs are active during one stage and inactive during other stages in the analysis.

The effect of variable elastic modulus in different soil layers can be taken into account in this paper to analyse retaining structures. In fact, many researchers have proposed different methods. The method adopted in our paper is as follows. For different soil layers with variable soil elastic modulus $m_{i}$, the mean value $m$ of the elastic modulus for the whole soil domain can be taken as the weighted average value of the soil elastic modulus and thickness of each layer. That is:

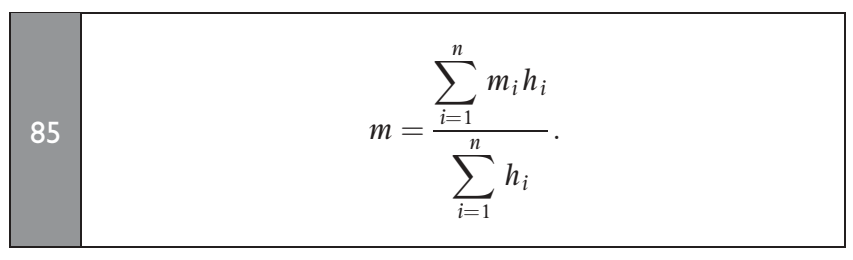

The large deformation cases really exist for retaining structures. It is thus necessary to take into account the elasticplastic behaviour of soil when conducting the failure analysis of retaining structures. However, the objective of this study is to propose a simple and practical procedure for the analysis of multi-braced retaining structures, taking into account various excavation stages. We have therefore not considered the nonlinear behaviour of soil in this paper. We shall study the nonlinear effects of soil in the next phase of our study by adopting a proper non-linear soil model and using Gleser's approximation method in the analysis procedure.

As mentioned above, we have conducted many site measurements of deformation of retaining structures in Shanghai. Comparisons were made between the results from the proposed method and the measured data, and satisfactory agreement has been found. The accuracy of the proposed method has also been verified by other methods (e.g. the finiteelement method). It is noted that the largest deformation was usually found near the excavation line, which has been proved by many site measurements from various projects. Owing to limitations of construction site conditions, we have not yet conducted site measurement of the bending moment and shear force of retaining structures. However, based on the principles of structural mechanics, the computational results for bending moment and shear force may agree well with the test data as long as the calculated deformation results are in close agreement with the measured data.

\section{REFERENCES}

11. Bowles J. E. Foundation Analysis and Design, 5th edn. McGraw-Hill, New York, 1996.

12. PotTs D. M. 42nd Rankine Lecture. Numerical analysis: a virtual dream or practical reality? Géotechnique, 2003, 53, No. 6, 535-572.

13. SPANGLeR M. G. and MiCKLE J. Later pressure on retaining walls due to backfill surface loads. HRB Bulletin, 1956, No. $141,1-18$.

Please email, fax or post your discussion contributions to the secretary by I April 2005: email: daniela.wong@ice.org.uk; fax: +44 (0)20 665 2294; or post to Daniela Wong, Journals Department, Institution of Civil Engineers, I-7 Great George Street, London SWIP 3AA. 